\title{
Phosphine-Free Cross-Coupling Reaction of Arylboronic Acids with Carboxylic anhydrides or Acyl Chlorides in Aqueous Media
}

\author{
Bingwei Xin ${ }^{\dagger}{ }^{\ddagger}$ Yuhong Zhang, ${ }^{\dagger * *}$ Kai Cheng ${ }^{\dagger}$ \\ ${ }^{\dagger}$ Department of Chemistry, Zhejiang University, Hangzhou 310027, P.R. China. \\ ${ }^{\ddagger}$ Department of Chemistry, Dezhou University, Dezhou 253023, P.R. China \\ E-mail: yhzhang@zjuem.zju.edu.cn
}

Supporting Information

\section{Content}

General procedure for the acylation reaction...............S2

Characterization data of the ketones........................S4

Reference............................................ 21 
General Starting materials and solvents were purchased from common commercial sources and were used without additional purification. ${ }^{1} \mathrm{H}$ NMR spectra were recorded at 500 or $400 \mathrm{MHz}$, using TMS as internal standard. Mass spectroscopy data of the product of acylation reaction was collected on a MS-EI instrument. 1-Butyl-3-methylimidazoliumchloride [bmim][Cl], 1-butyl-3-methylimidazolium tetrafluoroborate $\left([\mathrm{bmim}]\left[\mathrm{BF}_{4}\right]\right), \quad$ and 1-butyl-3-methylimidazolium hexafluorophosphate $\left([\mathrm{bmim}]\left[\mathrm{PF}_{6}\right]\right)$ ionic liquids were prepared according to the procedure reported previously in the literature. ${ }^{1}$

[Bmim][Cl]: ${ }^{1} \mathrm{H}$ NMR $\left(500 \mathrm{MHz}, \mathrm{D}_{2} \mathrm{O}\right): \delta 9.01(\mathrm{~s}, 1 \mathrm{H}) ; 7.74(\mathrm{~d}, 1 \mathrm{H}, J=2.2 \mathrm{~Hz})$; $7.69(\mathrm{~d}, 1 \mathrm{H}, J=2.2 \mathrm{~Hz}) ; 4.33(\mathrm{t}, 2 \mathrm{H}, J=9.3 \mathrm{~Hz}) ; 4.02(\mathrm{~s}, 3 \mathrm{H}) ; 1.90(\mathrm{~m}, 2 \mathrm{H}) ; 1.36(\mathrm{~m}$, $2 \mathrm{H}) ; 0.93(\mathrm{t}, 3 \mathrm{H}, J=9.1 \mathrm{~Hz})$.

[Bmim][BF 4 ]: ${ }^{1} \mathrm{H}$ NMR $\left(500 \mathrm{MHz}, \mathrm{CD}_{3} \mathrm{COCD}_{3}\right): \delta 8.99(\mathrm{~s}, 1 \mathrm{H}) ; 7.75(\mathrm{~d}, 1 \mathrm{H}, J=2.2$ $\mathrm{Hz}) ; 7.70(\mathrm{~d}, 1 \mathrm{H}, J=2.2 \mathrm{~Hz}) ; 4.35(\mathrm{t}, 2 \mathrm{H}, J=9.3 \mathrm{~Hz}) ; 4.04(\mathrm{~s}, 3 \mathrm{H}) ; 1.91(\mathrm{~m}, 2 \mathrm{H}) ; 1.37$ (m, 2H); 0.94(t, 3H, $J=9.1 \mathrm{~Hz})$.

[Bmim][PF 6 ]: ${ }^{1} \mathrm{H}$ NMR (500 MHz, $\left.\mathrm{CD}_{3} \mathrm{COCD}_{3}\right): \delta 8.99(\mathrm{~s}, 1 \mathrm{H}) ; 7.76(\mathrm{~d}, 1 \mathrm{H}, J=2.2$ $\mathrm{Hz}) ; 7.71(\mathrm{~d}, 1 \mathrm{H}, J=2.2 \mathrm{~Hz}) ; 4.36(\mathrm{t}, 2 \mathrm{H}, J=9.3 \mathrm{~Hz}) ; 4.06(\mathrm{~s}, 3 \mathrm{H}) ; 1.93(\mathrm{~m}, 2 \mathrm{H}) ; 1.38$ $(\mathrm{m}, 2 \mathrm{H}) ; 0.94(\mathrm{t}, 3 \mathrm{H}, J=9.1 \mathrm{~Hz})$

General procedure for the acylation reaction in PEG2000: A round-bottomed flask was charged with a mixture of $\mathrm{Na}_{2} \mathrm{CO}_{3}(0.170 \mathrm{~g}, 1.6 \mathrm{mmol}), \mathrm{Pd}(\mathrm{OAc})_{2}(1 \mathrm{mg}$, $0.5 \mathrm{~mol} \%$ ), PEG2000 (3 g), and $\mathrm{H}_{2} \mathrm{O}(3 \mathrm{~g})$. The solution was heated to $60{ }^{\circ} \mathrm{C}$ with stirring, and carboxylic anhydride $(1 \mathrm{mmol})$ and arylboronic acid $(1.2 \mathrm{mmol})$ was added. The resulted suspension was stirred at $60{ }^{\circ} \mathrm{C}$ for the indicated time, and cooled to room temperature. The suspension was extracted with diethyl ether $(5 \mathrm{~mL})$ with four times. The combined ethyl ether phase was concentrated and the product was obtained by purification of flash chromatography on a silica gel column. In the recycle 
experiment, the residue after the extraction was subjected to a second run of the acylation reaction by charging with the same substrates (benzoic anhydride, phenylboronic acid and $\mathrm{Na}_{2} \mathrm{CO}_{3}$ ) without further addition of $\mathrm{Pd}(\mathrm{OAc})_{2}, \mathrm{PEG} 2000$ or water.

General procedure for the acylation reaction in $[\mathrm{bmim}]\left[\mathrm{PF}_{6}\right]$ : A round-bottomed flask was charged with a mixture of $\mathrm{Na}_{2} \mathrm{CO}_{3}(0.170 \mathrm{~g}, 1.6 \mathrm{mmol}), \mathrm{Pd}(\mathrm{OAc})_{2}(4 \mathrm{mg}$, $1.8 \mathrm{~mol} \%)$, [bmim] $\left[\mathrm{PF}_{6}\right](3 \mathrm{~g})$, and $\mathrm{H}_{2} \mathrm{O}(2.5 \mathrm{~g})$. The solution was heated to $60{ }^{\circ} \mathrm{C}$ with stirring, and carboxylic anhydride $(1 \mathrm{mmol})$ and arylboronic acid $(1.2 \mathrm{mmol})$ was added. The mixture was stirred at $60{ }^{\circ} \mathrm{C}$ for the indicated time and cooled to room temperature. The suspension was extracted with diethyl ether $(5 \mathrm{~mL})$ with four times. The combined ethyl ether phase was concentrated, and the further purification of the product was achieved by flash chromatography on a silica gel column. In the recycle experiment, the residue after the extraction was washed with water $(3 \times 6 \mathrm{~mL})$ and then was subjected to a second run of the acylation reaction by charging with the same substrates (benzoic anhydride, phenylboronic acid, water, and $\mathrm{Na}_{2} \mathrm{CO}_{3}$ ) without further addition of $\mathrm{Pd}(\mathrm{OAc})_{2}$ or IL. 


\section{Characterization data of the product of the Suzuki reaction}

1.<smiles>O=C(c1ccccc1)c1ccccc1</smiles>

$[\mathbf{T 1}, 119-61-9$, Ref .2]

Benzophenone: ${ }^{1} \mathrm{H}$ NMR (500 MHz, $\mathrm{CDCl}_{3}$, TMS): $\delta$ 7.82-7.81 (t, $J=4.1 \mathrm{~Hz}, 4 \mathrm{H}$ ), 7.61-7.59 (m, $2 \mathrm{H}), 7.51-7.48$ (t, $J=7.7 \mathrm{~Hz}, 4 \mathrm{H}) . \mathrm{MS}(\mathrm{EI}): \mathrm{m} / \mathrm{z}(\%): 182(75)\left[\mathrm{M}^{+}\right]$, 105 (100), 77 (56), 51(15).
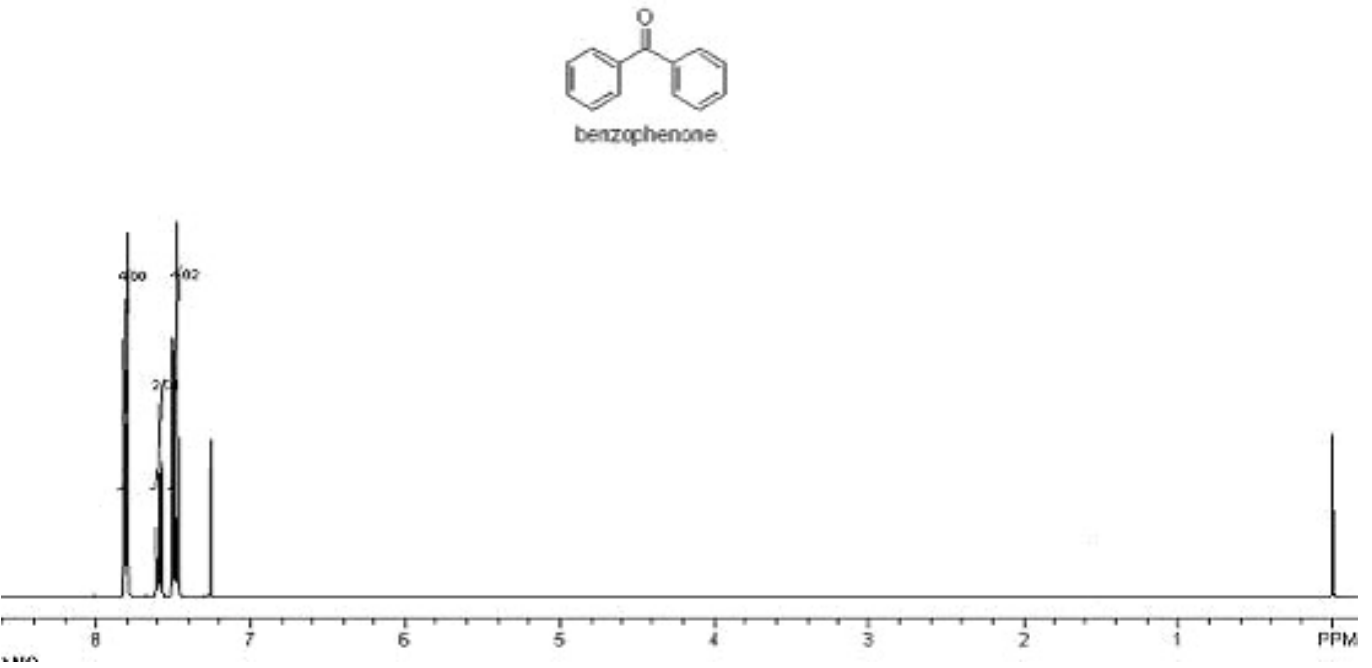
2.<smiles>Cc1ccc(C(=O)c2ccccc2)cc1</smiles>

[T2-2, 134-84-9, Ref .3]

4-Methylbenophenone: ${ }^{1} \mathrm{H}$ NMR (500 MHz, $\mathrm{CDCl}_{3}$, TMS): $\delta$ 7.68-7.66 (t, $J=4.2 \mathrm{~Hz}$, $2 \mathrm{H}), 7.62-7.60$ (d, $J=8.1 \mathrm{~Hz}, 2 \mathrm{H}), 7.47-7.44$ (t, $J=7.4 \mathrm{~Hz}, 1 \mathrm{H}), 7.37-7.34$ (t, $J=7.6$ Hz, 2 H), 7.17-7.15 (d, J=8.0 Hz, 2 H), 2.32 (s, 3 H). MS (EI): m/z (\%): 196(M $\left.{ }^{+}, 60\right)$, 181(15), 165(5), 152(5), 119(100), 105(60), 91(40), 77(40), 65(20), 51(15).

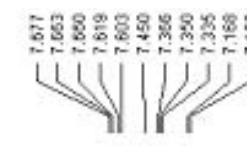

COMAENT: Dhejiang University Axance DMN 600 ONP Emen Single xtrm-2-27-16 in CDCL3
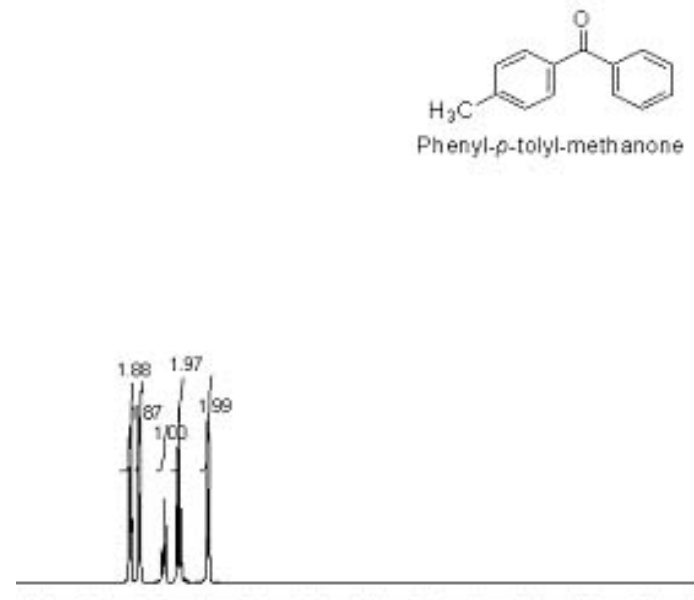

$\ldots$

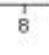


3.<smiles>COc1ccc(C(=O)c2ccccc2)cc1</smiles>

[T2-3, 611-94-9, Ref .4]

4-Methoxybenzophenone: ${ }^{1} \mathrm{H}$ NMR (500 MHz, $\left.\mathrm{CDCl}_{3}, \mathrm{TMS}\right): \delta$ 7.84-7.82 (m, $\left.2 \mathrm{H}\right)$, 7.76-7.74 (t, $J=4.2 \mathrm{~Hz}, 2 \mathrm{H}), 7.56-7.54(\mathrm{~d}, J=7.5 \mathrm{~Hz}, 1 \mathrm{H}), 7.48-7.45$ (t, $J=7.6 \mathrm{~Hz}, 2$ H), 6.97-6.95 (d, J=8.8 Hz, 2 H), 3.87 (s, 3 H). MS (EI): m/z (\%): 212 (70) [M $\left.{ }^{+}\right], 181$ (3), 135 (100), 105(10), 92 (12), 77 (28), 51(6).
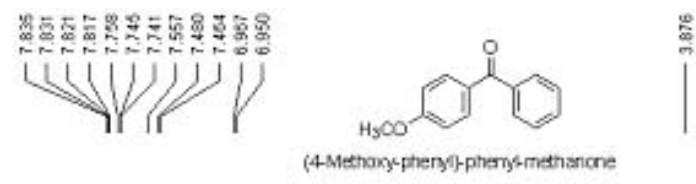

COMWENT: Thejisng Unweraity toance DWK 500 , ONP $5 \mathrm{~mm}$ Sample. $x \mathrm{bm}-2 \cdot 20-3$ in $\mathrm{CDCL} 3$

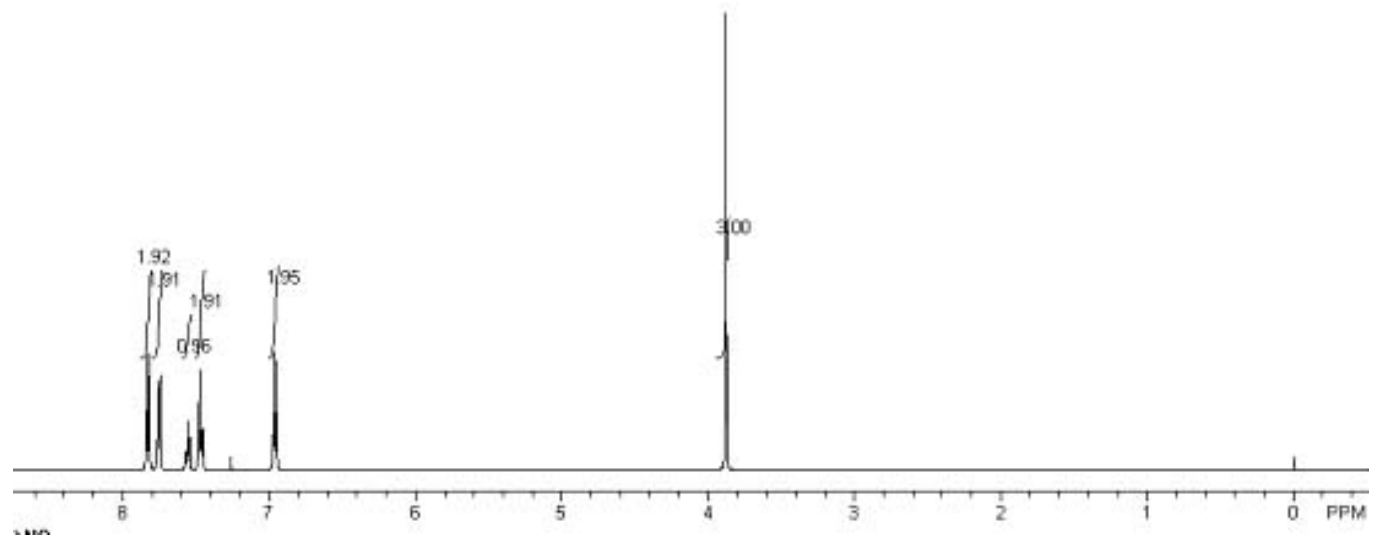


4.<smiles>O=C(c1ccccc1)c1ccc(C(F)(F)F)cc1</smiles>

[T2-4, 728-86-9, Ref .5]

4-Trifluoromethylbenzophenone: ${ }^{1} \mathrm{H}$ NMR (500 MHz, $\left.\mathrm{CDCl}_{3}, \mathrm{TMS}\right): \delta$ 7.90-7.89 (d, $J$ $=7.3 \mathrm{~Hz}, 2 \mathrm{H}), 7.81-7.80(\mathrm{t}, J=8.1 \mathrm{~Hz}, 2 \mathrm{H}), 7.76-7.75(\mathrm{~d}, J=8.1 \mathrm{~Hz}, 2 \mathrm{H}), 7.65-7.61$ (m, $1 \mathrm{H}), 7.52-7.49$ (q, $J=7.2 \mathrm{~Hz}, 2 \mathrm{H}) . \mathrm{MS}(\mathrm{EI}): \mathrm{m} / \mathrm{z}(\%): 250(60)\left[\mathrm{M}^{+}\right], 181$ (7), 173 (100), 105(30), 77 (40).

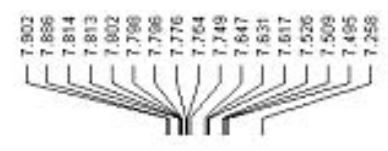

\section{Theirang Uhiversty Awance DMX 500 ,} CNP Srmm Samele:xbr: 2.20 .4 in CDOL
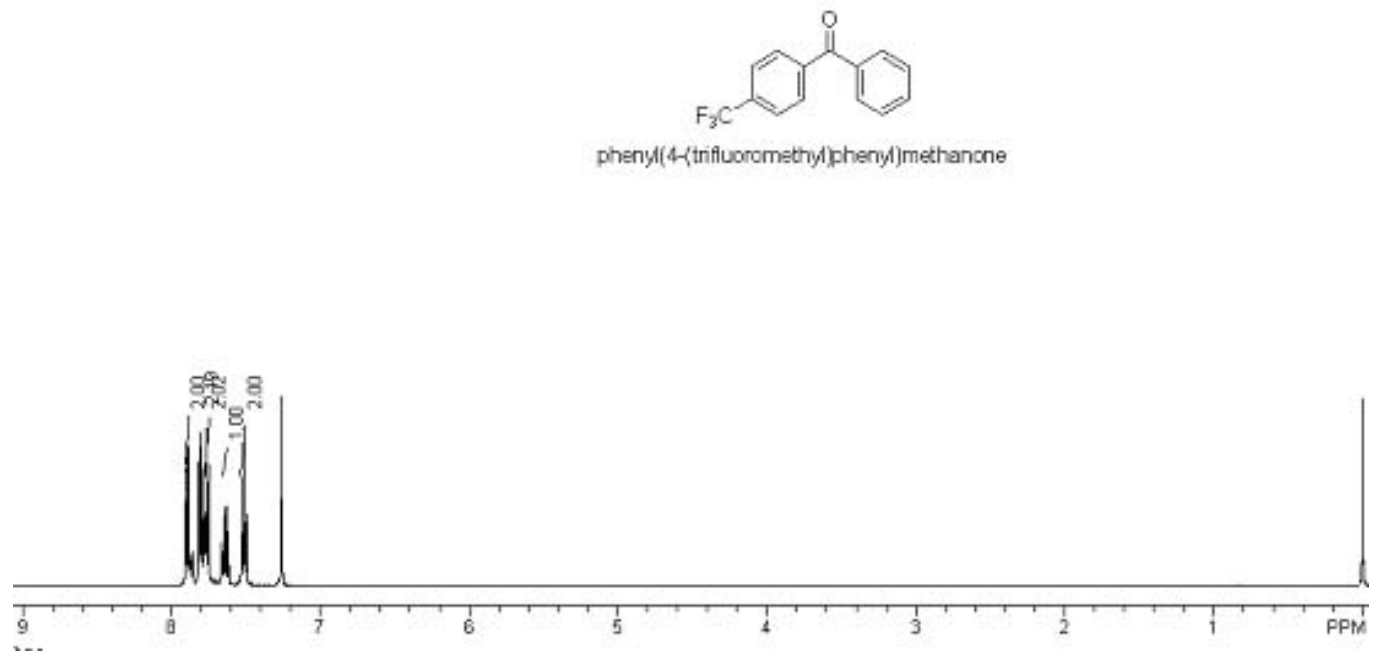
5.<smiles>O=C(c1ccccc1)c1ccc2ccccc2c1</smiles>

[T2-5, 644-13-3, Ref .6]

2-Benzoylnaphthane: ${ }^{1} \mathrm{H}$ NMR (400 MHz, $\mathrm{CDCl}_{3}$, TMS): $\delta 8.27$ (s, $\left.1 \mathrm{H}\right), 7.96$ (s, $2 \mathrm{H}$ ), 7.94-7.92 (d, $J=7.6 \mathrm{~Hz}, 2 \mathrm{H}), 7.88-7.86(\mathrm{~d}, J=6.8 \mathrm{~Hz}, 2 \mathrm{H}), 7.64-7.61(\mathrm{~m}, 2 \mathrm{H})$, 7.59-7.51(m, 3 H). MS (EI): m/z (\%): 233(M+1 $\left.{ }^{+}, 10\right), 232\left(\mathrm{M}^{+}, 60\right), 155(100), 127(80)$, 105(40), 77(75), 51(20).
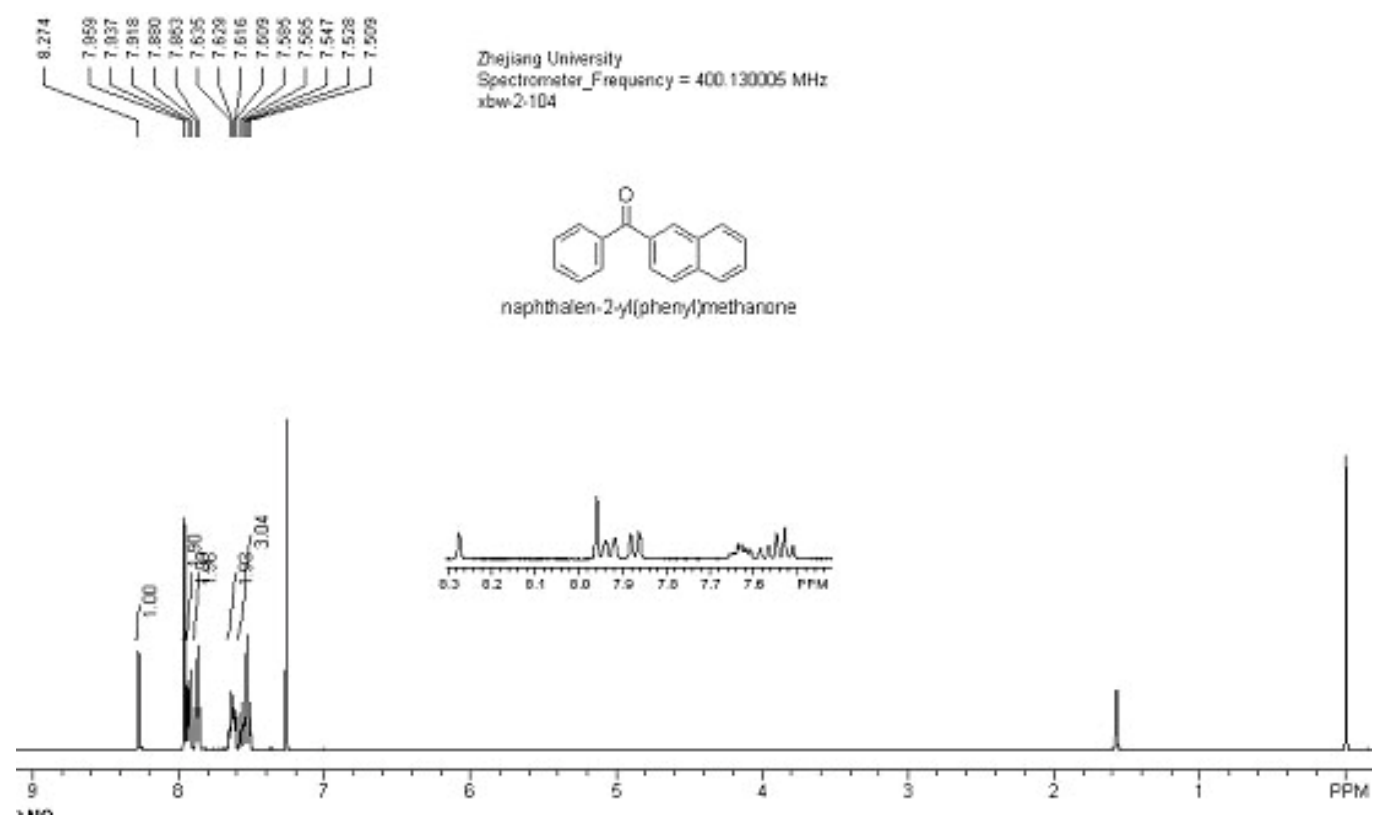
6.<smiles>O=C(c1ccccc1)c1cccc2ccccc12</smiles>

[T2-6, 642-29-5, Ref .7]

1-Benzoylnaphthane: ${ }^{1} \mathrm{H}$ NMR (400 MHz, $\mathrm{CDCl}_{3}$, TMS): $\delta 8.11-8.09$ (d, $J=8.0 \mathrm{~Hz}, 1$ H), 8.02-8.00 (d, $J=8.0 \mathrm{~Hz}, 1 \mathrm{H}), 7.94-7.92$ (t, $J=4.8 \mathrm{~Hz}, 1 \mathrm{H}), 7.89-7.86$ (q, $J=3.3$ Hz, 2 H), 7.63-7.56 (m, 2H), 7.56-7.51 (m, 3H), 7.51-7.45 (m, 2H). MS (EI): m/z (\%): 233(M+1 $\left.{ }^{+}, 12\right), 232\left(\mathrm{M}^{+}, 60\right), 155(100), 127(60), 105(25), 77(40), 51(20)$.
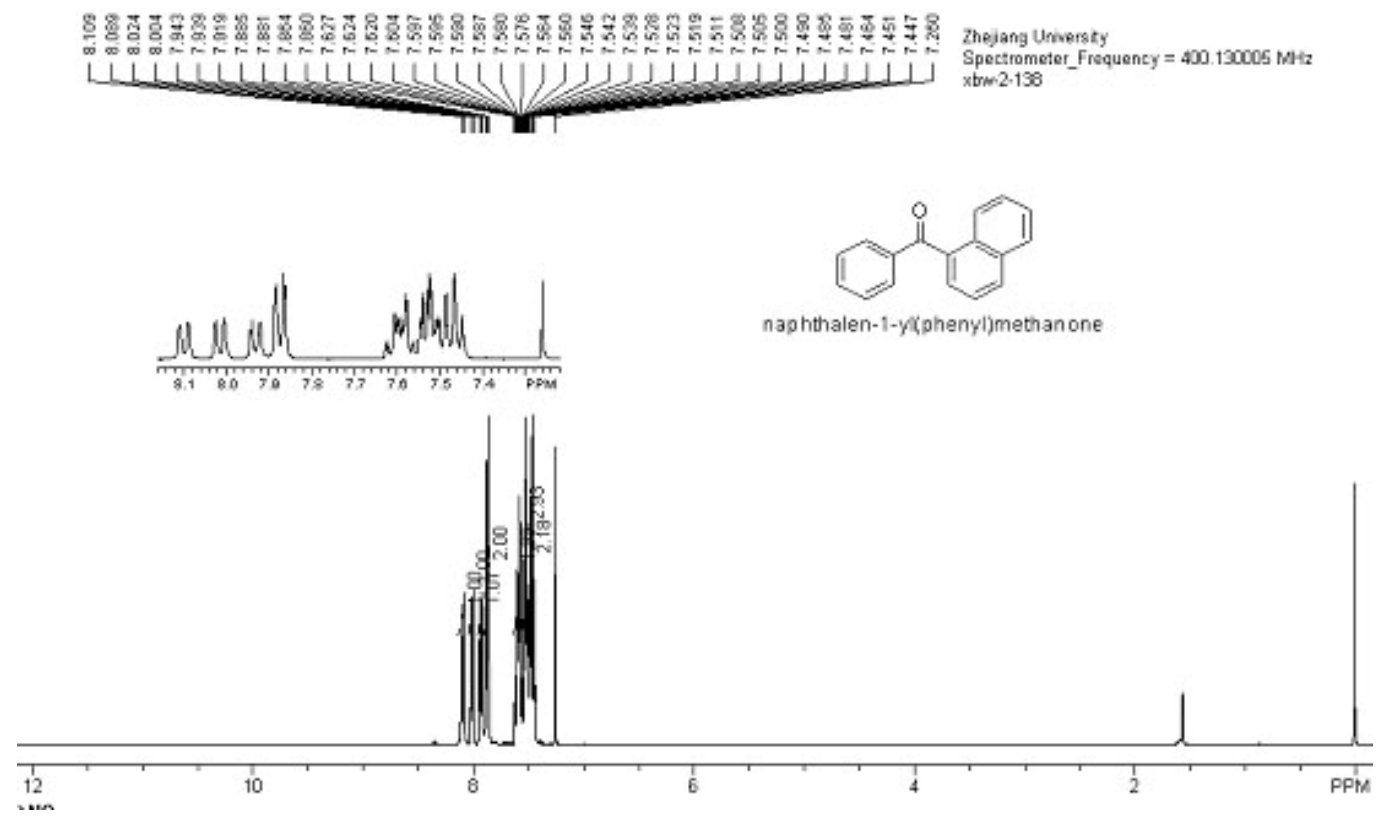
7.

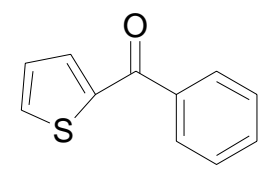

[T2-7, 135-00-2, Ref .8]

2-Benzoylthiophene: ${ }^{1} \mathrm{H}$ NMR (400 $\left.\mathrm{MHz}, \mathrm{CDCl}_{3}, \mathrm{TMS}\right): \delta$ 7.87-7.85 (m, $2 \mathrm{H}$ ), 7.72-7.71 (q, $J=2.0 \mathrm{~Hz}, 1 \mathrm{H}), 7.65-7.63(\mathrm{q}, J=1.7 \mathrm{~Hz}, 1 \mathrm{H}), 7.61-7.57(\mathrm{~m}, 1 \mathrm{H})$,

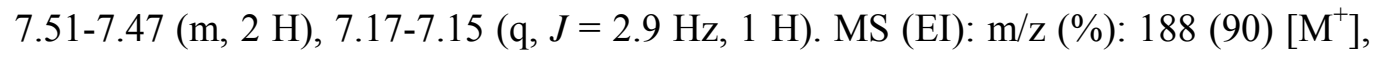
171 (10), 160(10), 111 (100), 105(40), 77 (20), 51(12).

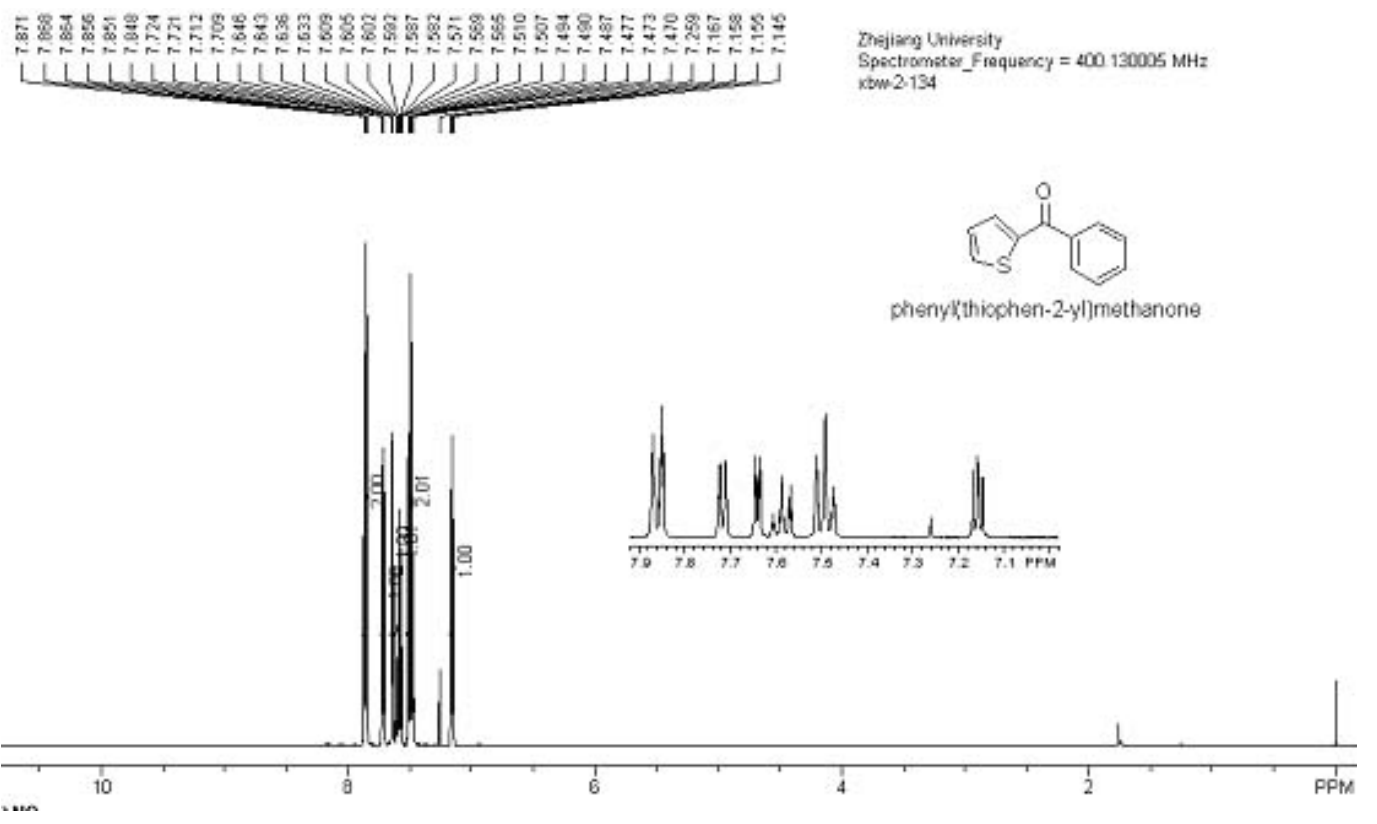


8.

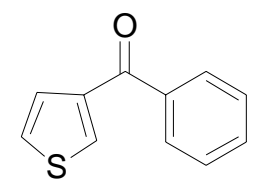

[T2-8, 6453-99-2, Ref .9]

3-Benzoylthiophene: ${ }^{1} \mathrm{H}$ NMR (400 MHz, $\mathrm{CDCl}_{3}$, TMS): $\delta$ 7.87-7.85 (t, $J=4.2 \mathrm{~Hz}, 2$ H), 7.73-7.71 (m, $1 \mathrm{H})$, 7.65-7.63 (m, $1 \mathrm{H})$, 7.61-7.57 (m, $1 \mathrm{H})$, 7.51-7.47 (m, $2 \mathrm{H})$, 7.17-7.14 (m, 1 H). MS (EI): m/z (\%): $188(95)\left[\mathrm{M}^{+}\right], 171$ (10), 160(10), 111 (100), 105(40), 77 (20), 51(12), 39(12).

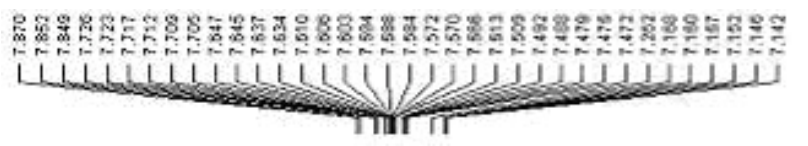

Dojeing Uhimersity Speclrometer_fiequency $=200.150005 \mathrm{hHz}$ stem-2.136
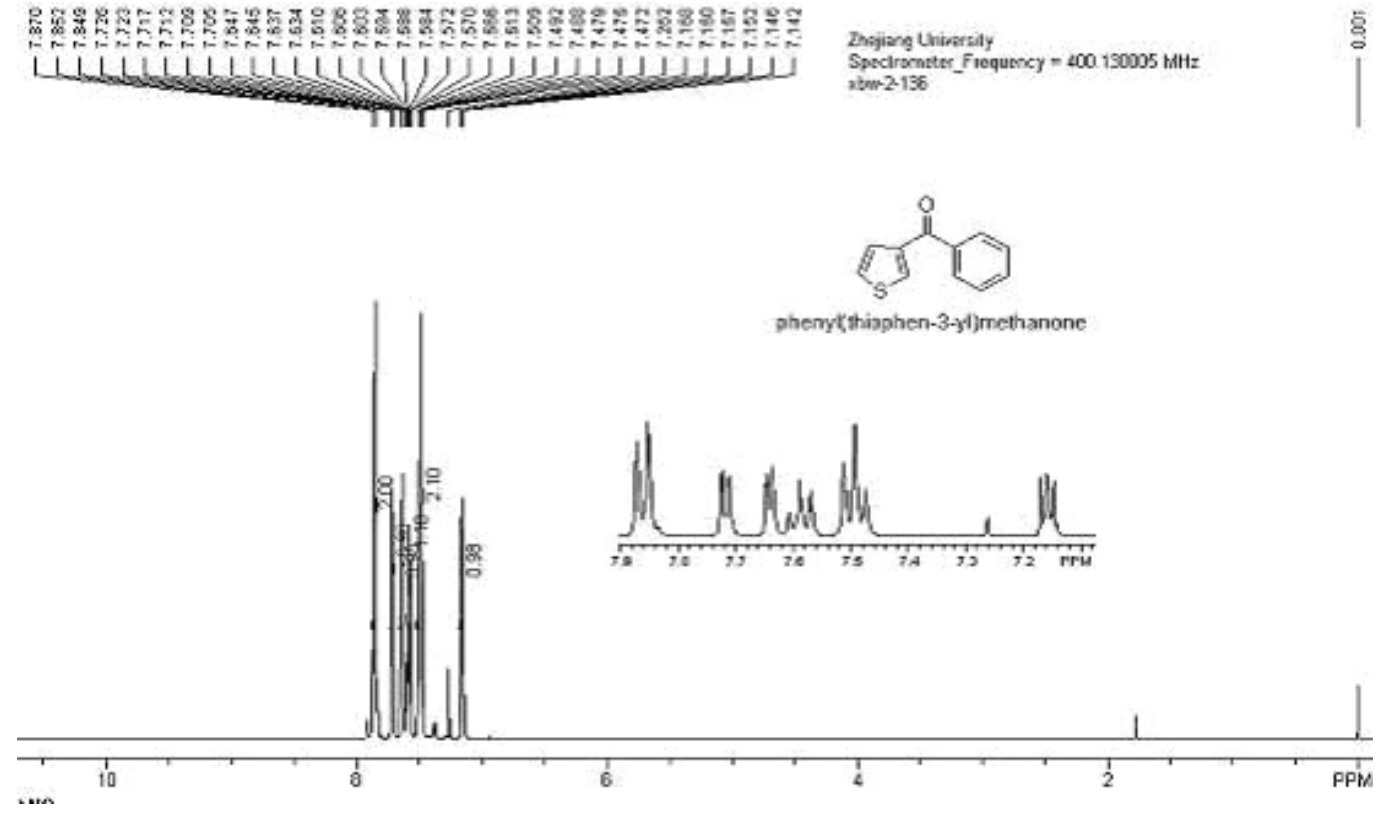
9.

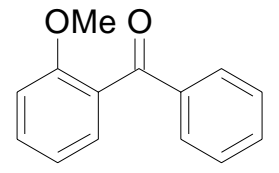

[T2-11, 2553-04-0, Ref .10]

2-Methoxybenzophenone: ${ }^{1} \mathrm{H}$ NMR (400 MHz, $\mathrm{CDCl}_{3}$, TMS): $\delta$ 7.83-7.80 (q, $J=3.1$ $\mathrm{Hz}, 2 \mathrm{H}), 7.58-7.54$ (t, $J=7.2 \mathrm{~Hz}, 1 \mathrm{H}), 7.45-7.43$ (m, $3 \mathrm{H}), 7.37-7.35$ (q, $J=2.4 \mathrm{~Hz}, 1$ H), 7.04-6.98 (m, 2 H), 3.73 (s, 3 H). MS (EI): m/z (\%): 212 (60) [M+1, 195(30), 181 (10), 135 (100), 121(20), 105(30), 92 (18), 77 (70), 51(25).

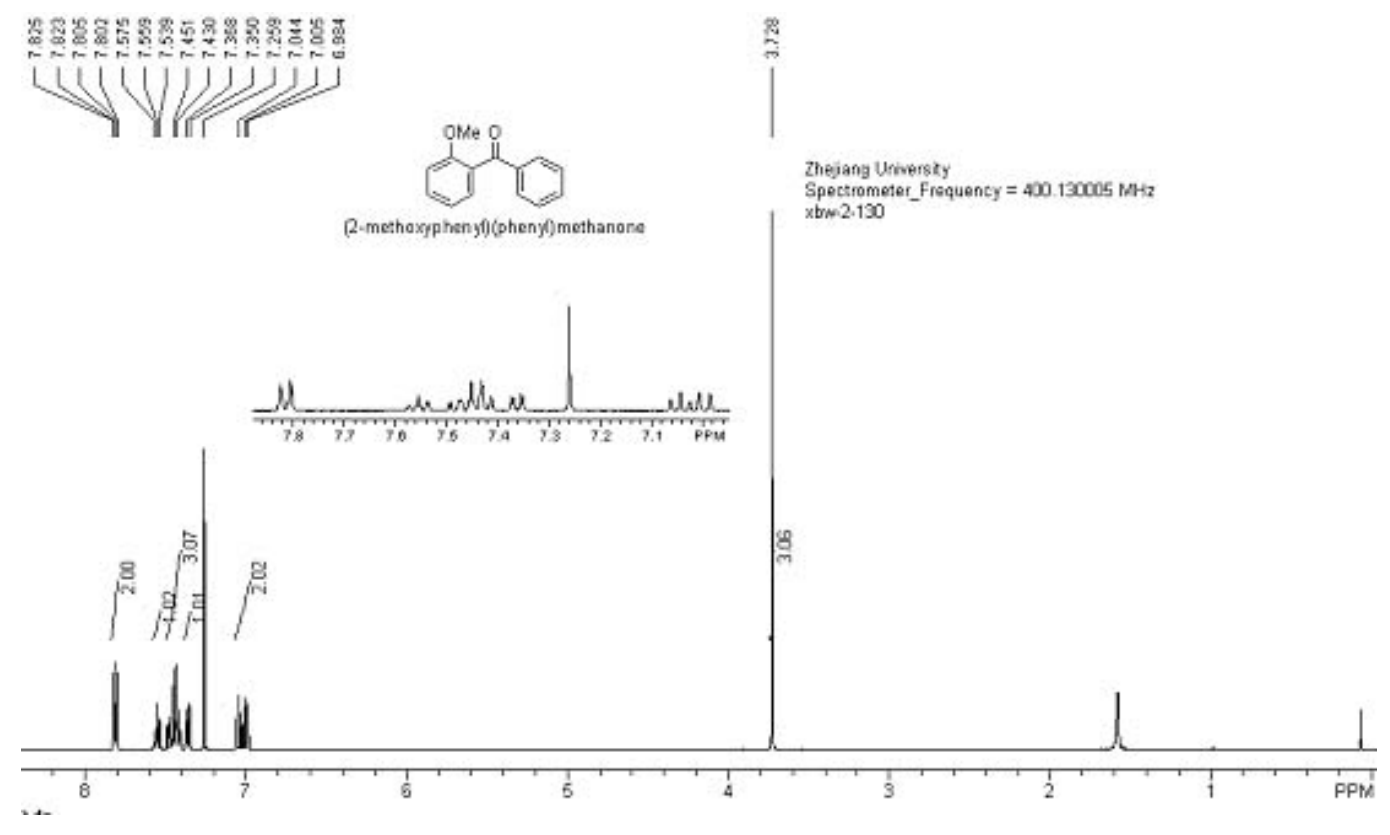


10.<smiles>O=C(c1ccccc1)c1ccc(Cl)cc1</smiles>

[T2-12, 134-85-0, Ref .10]

4-Chlorobenzophenone: ${ }^{1} \mathrm{H}$ NMR (500 MHz, $\left.\mathrm{CDCl}_{3}, \mathrm{TMS}\right): \delta$ 7.78-7.75 (m, $4 \mathrm{H}$ ), 7.62-7.59 (m, $1 \mathrm{H})$, 7.51-7.46 (m, $4 \mathrm{H}) . \mathrm{MS}(\mathrm{EI}): \mathrm{m} / \mathrm{z}(\%): 218(100)\left(\mathrm{M}^{+},{ }^{37} \mathrm{Cl}, 10\right)$, $216\left(\mathrm{M}^{+},{ }^{35} \mathrm{Cl}, 30\right), 181$ (10), 139(50), $111(50), 105$ (80), 77(100), 51(70).

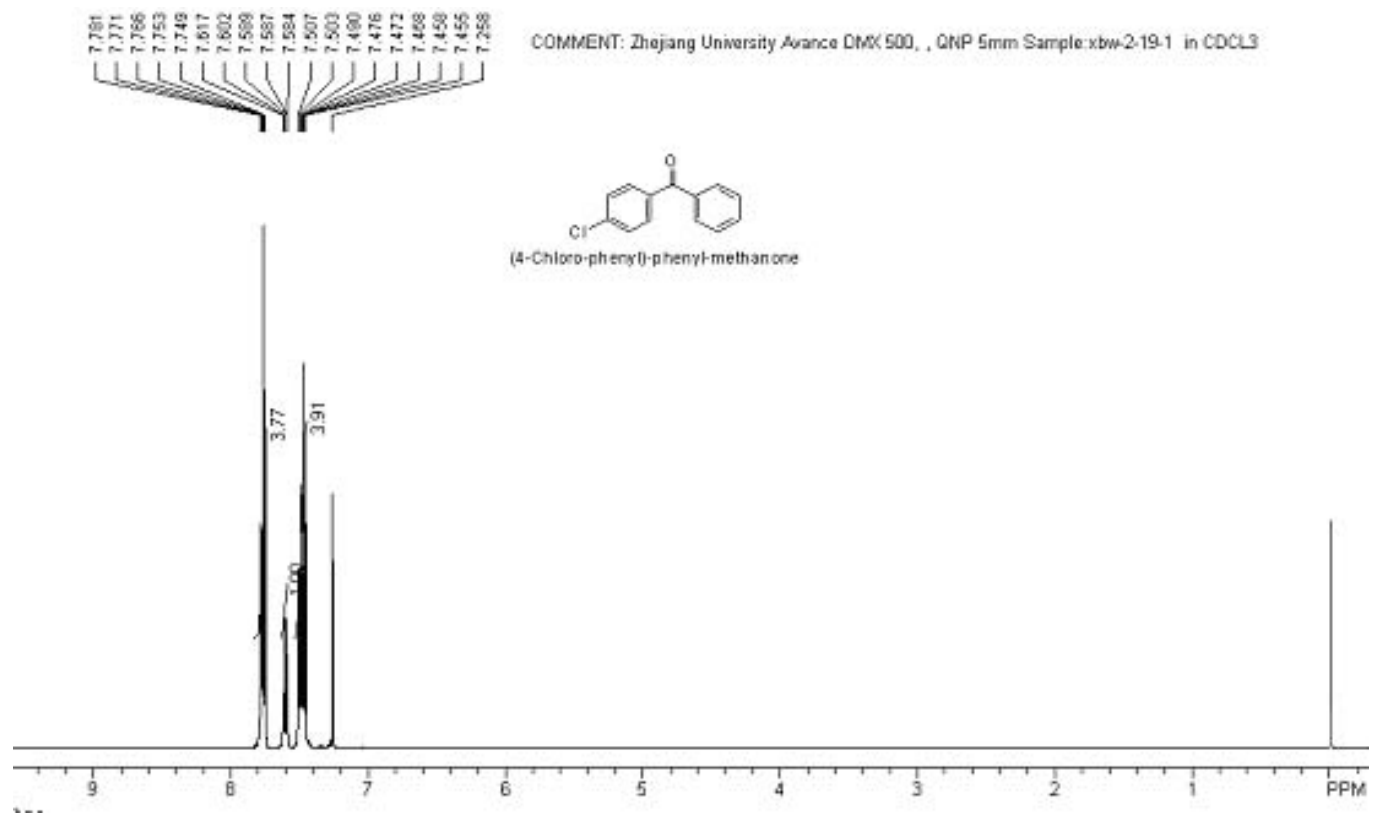


11.<smiles>O=C(c1ccccc1)c1cccc(Cl)c1</smiles>

[T2-13, 1016-78-0, Ref .11]

3-Chlorobenzophenone: ${ }^{1} \mathrm{H}$ NMR (500 MHz, $\left.\mathrm{CDCl}_{3}, \mathrm{TMS}\right): \delta 7.81-7.79(\mathrm{t}, J=4.2 \mathrm{~Hz}$, $3 \mathrm{H}), 7.68-7.63(\mathrm{t}, J=9.3 \mathrm{~Hz}, 1 \mathrm{H}), 7.62-7.56(\mathrm{~m}, 2 \mathrm{H}), 7.52-7.49(\mathrm{t}, J=7.7 \mathrm{~Hz}, 2 \mathrm{H})$, 7.45-7.41 (t, $J=7.8 \mathrm{~Hz}, 1 \mathrm{H}) . \mathrm{MS}(\mathrm{EI}): \mathrm{m} / \mathrm{z}(\%): 218\left(\mathrm{M}^{+},{ }^{37} \mathrm{Cl}, 15\right), 216\left(\mathrm{M}^{+},{ }^{35} \mathrm{Cl}, 45\right)$, 181 (20), 139(50), 111 (100), 105 (30), 77(80), 51(50).
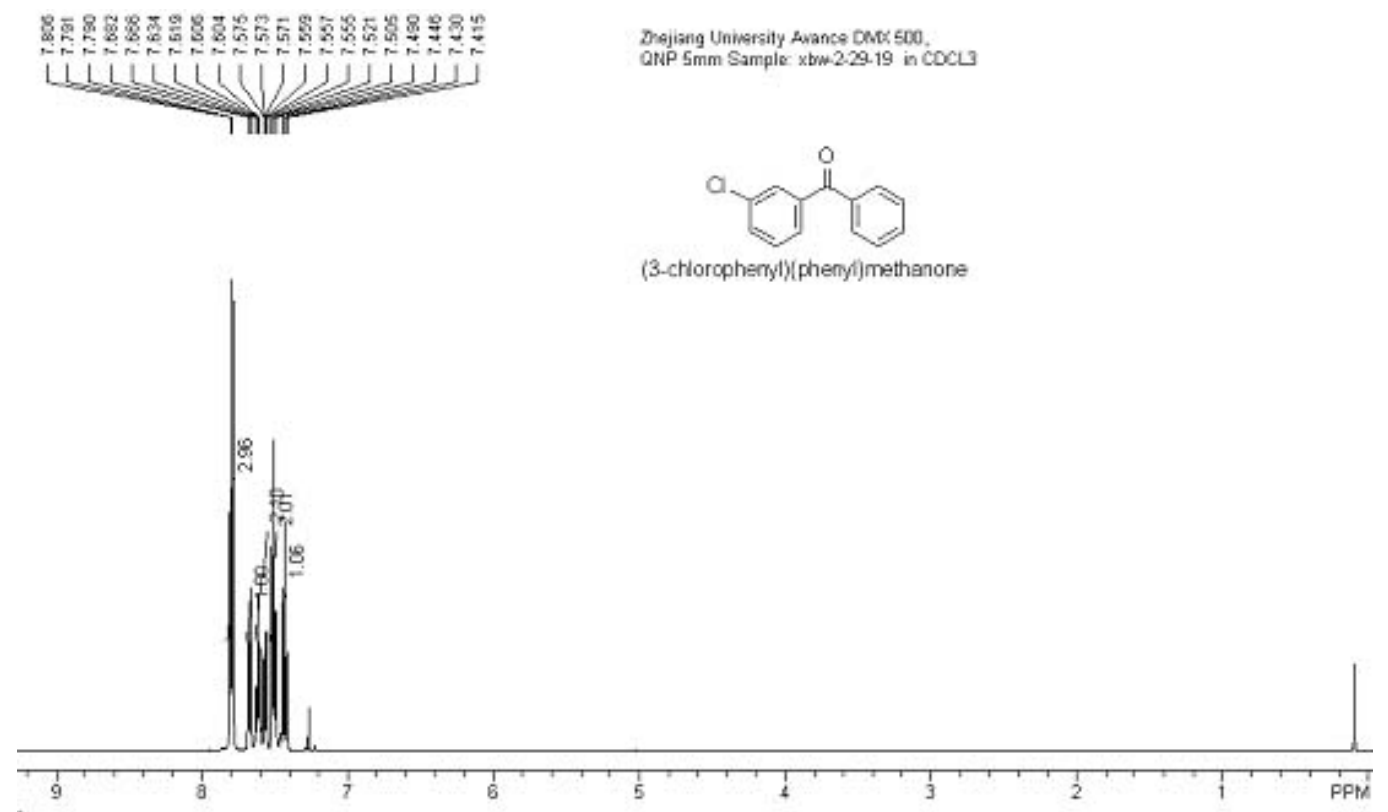

Dhejiang Uningrsity Avance Danx 500

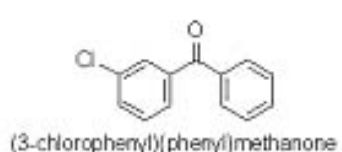


12.<smiles>O=C(c1ccccc1)c1ccccc1Cl</smiles>

[T2-14, 5162-03-8, Ref .12]

2-Chlorobenzophenone ${ }^{1} \mathrm{H}$ NMR (500 MHz, $\mathrm{CDCl}_{3}$, TMS): $\delta$ 7.75-7.73 (t, $J=4.2 \mathrm{~Hz}$, $2 \mathrm{H}), 7.54-7.51(\mathrm{t}, J=7.4 \mathrm{~Hz}, 1 \mathrm{H}), 7.41-7.37(\mathrm{~m}, 4 \mathrm{H}), 7.30-7.29(\mathrm{t}, J=4.3 \mathrm{~Hz}, 2 \mathrm{H})$. MS (EI): m/z (\%): $218\left(\mathrm{M}^{+},{ }^{37} \mathrm{Cl}, 15\right), 216\left(\mathrm{M}^{+},{ }^{35} \mathrm{Cl}, 45\right), 181$ (20), 139(50), 111 (50), 105 (80), 77(100), 51(70).
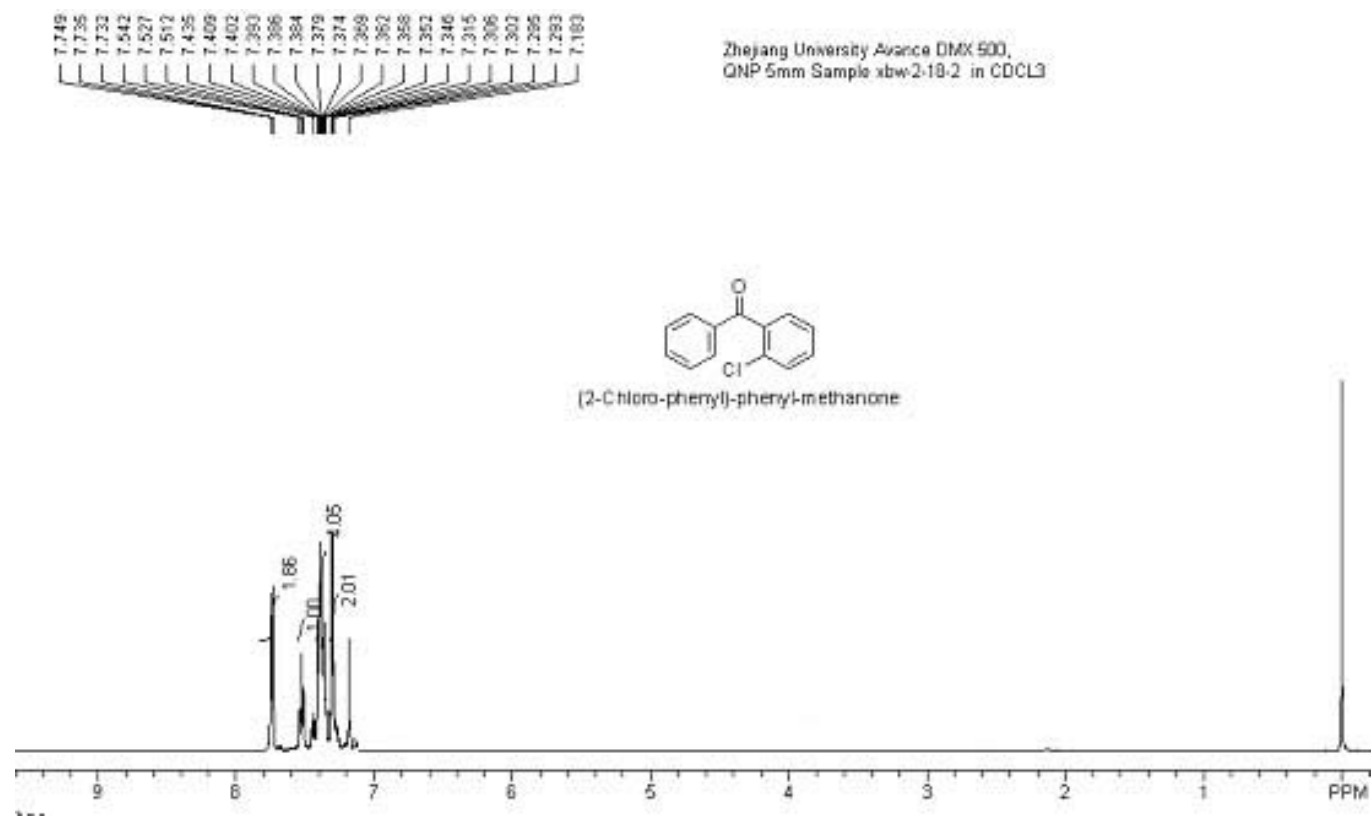
13.<smiles>O=C(c1ccccc1)c1ccc([N+](=O)[O-])cc1</smiles>

[T2-15, 1144-74-7, Ref .10]

4-Nitrobenzophenone: ${ }^{1} \mathrm{H}$ NMR (400 MHz, $\mathrm{CDCl}_{3}, \mathrm{TMS}$ ): $\delta 8.36-8.34$ (q, $J=2.9 \mathrm{~Hz}$, $2 \mathrm{H}), 7.95-7.93(\mathrm{q}, J=2.9 \mathrm{~Hz}, 2 \mathrm{H}), 7.81-7.79(\mathrm{t}, J=4.0 \mathrm{~Hz}, 2 \mathrm{H}), 7.66-7.64(\mathrm{~d}, J=8.0$ $\mathrm{Hz}, 1 \mathrm{H}), 7.55-7.51(\mathrm{t}, J=8.0 \mathrm{~Hz}, 2 \mathrm{H}) . \mathrm{MS}(\mathrm{EI}): \mathrm{m} / \mathrm{z}(\%): 227\left(\mathrm{M}^{+}, 75\right), 181(5)$, 150(20), 105(100), 77(60), 76(20), 51(20).

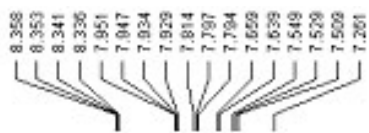

QATE: OCt 25 17:10:192005 Spoctromater frosuancy $=400.130005 \mathrm{MHA}$ XEW:265:2
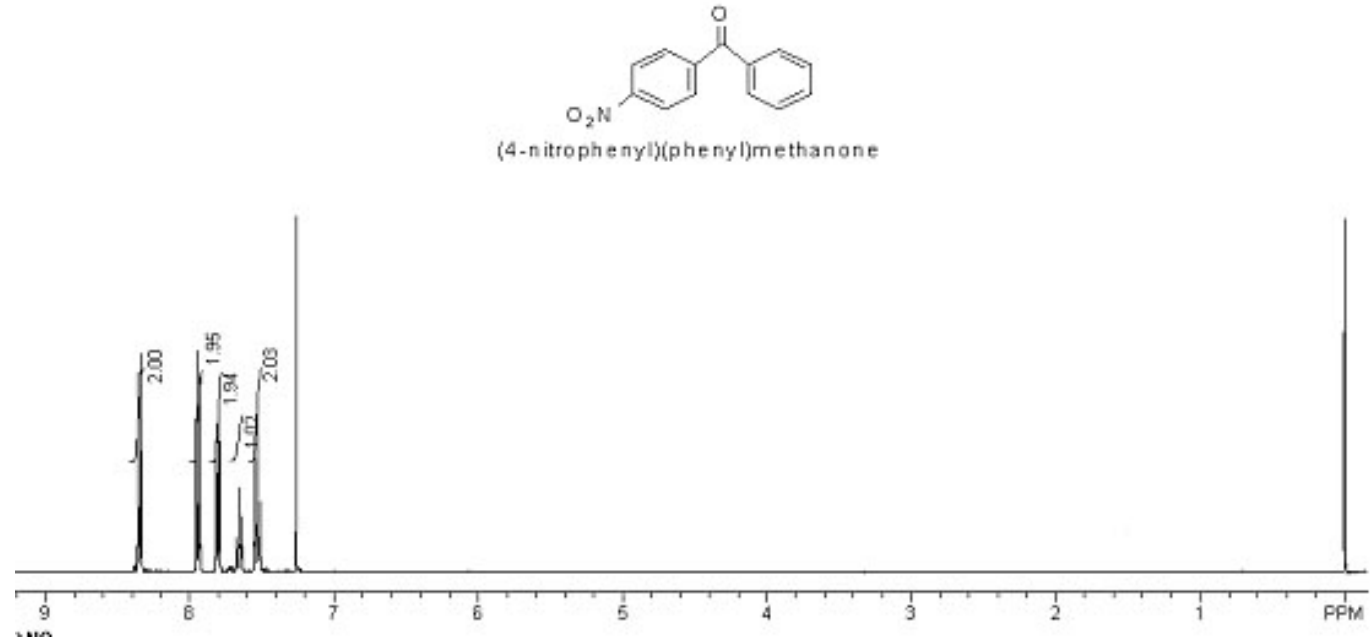
14.<smiles>N#Cc1ccc(C(=O)c2ccccc2)cc1</smiles>

[T2-16, 1503-49-7, Ref .13]

4-Benzoylbenzonitrile: ${ }^{1} \mathrm{H}$ NMR (400 MHz, $\mathrm{CDCl}_{3}$, TMS): $\delta$ 7.89-7.87 (t, $J=4.2 \mathrm{~Hz}$, $2 \mathrm{H}), 7.81-7.78(\mathrm{~m}, 4 \mathrm{H}), 7.67-7.63(\mathrm{~m}, 1 \mathrm{H}), 7.54-7.50(\mathrm{t}, J=7.6 \mathrm{~Hz}, 2 \mathrm{H})$. MS (EI): m/z (\%): 207 (40) $\left[\mathrm{M}^{+}\right], 130(20), 105$ (100), 77 (35), 51(18).
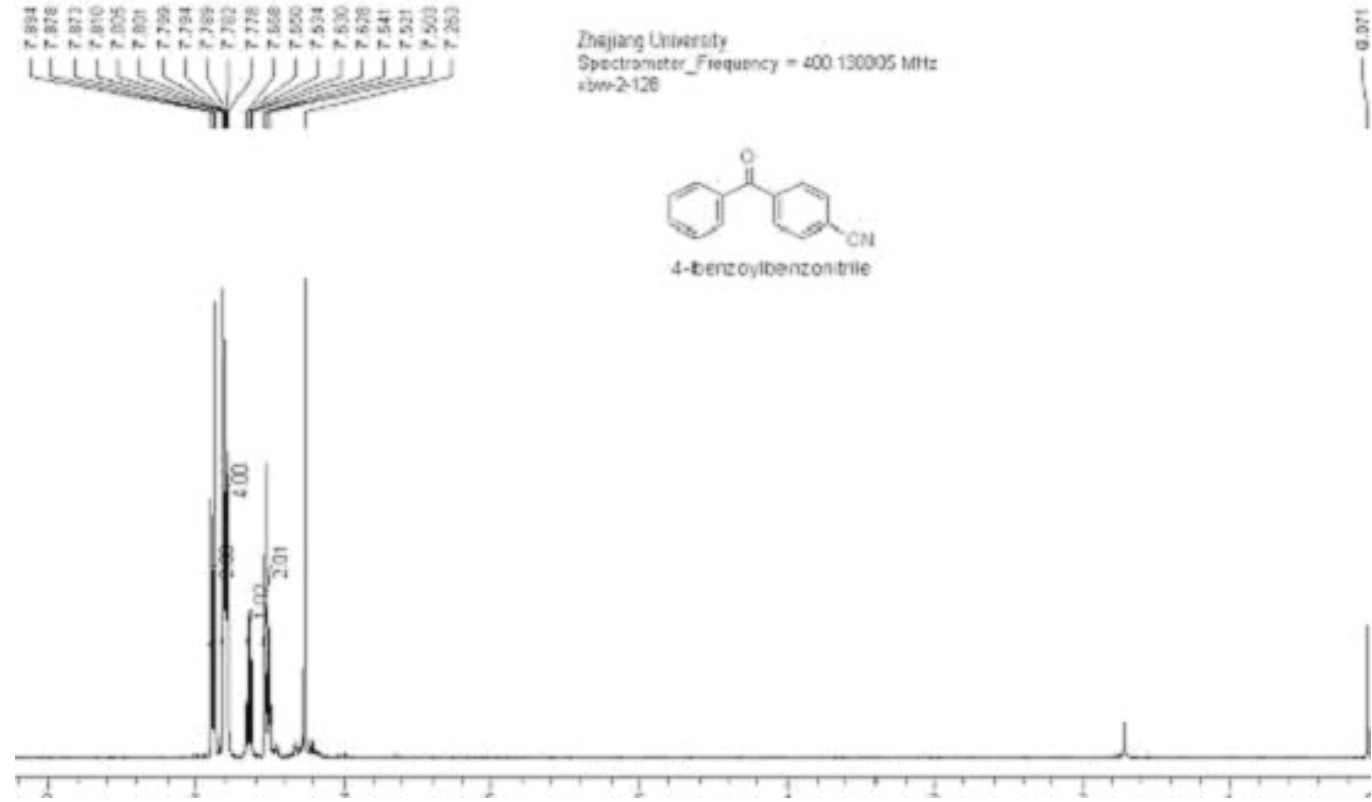
15.<smiles>O=C(c1ccccc1)c1ccco1</smiles>

[T2-17, 2689-59-0, Ref .14]

2-Benzoylfuran: ${ }^{1} \mathrm{H}$ NMR (500MHz, $\left.\mathrm{CDCl}_{3}, \mathrm{TMS}\right)$ : $\delta 7.89-7.88(\mathrm{t}, J=4.2 \mathrm{~Hz}, 2 \mathrm{H}$ ), 7.63-7.62 (d, $J=0.8 \mathrm{~Hz}, 1 \mathrm{H}), 7.53-7.49(\mathrm{~m}, 1 \mathrm{H}), 7.43-7.40(\mathrm{t}, J=7.7 \mathrm{~Hz}, 2 \mathrm{H})$, 7.16-7.15 (d, $J=3.5 \mathrm{~Hz}, 1 \mathrm{H}), 6.52-6.51(\mathrm{~m}, 1 \mathrm{H}) . \mathrm{MS}(\mathrm{EI}): \mathrm{m} / \mathrm{z}(\%): 172\left(\mathrm{M}^{+}, 80\right)$, 105(100), 95(80), 77(60), 67(30), 51(10).
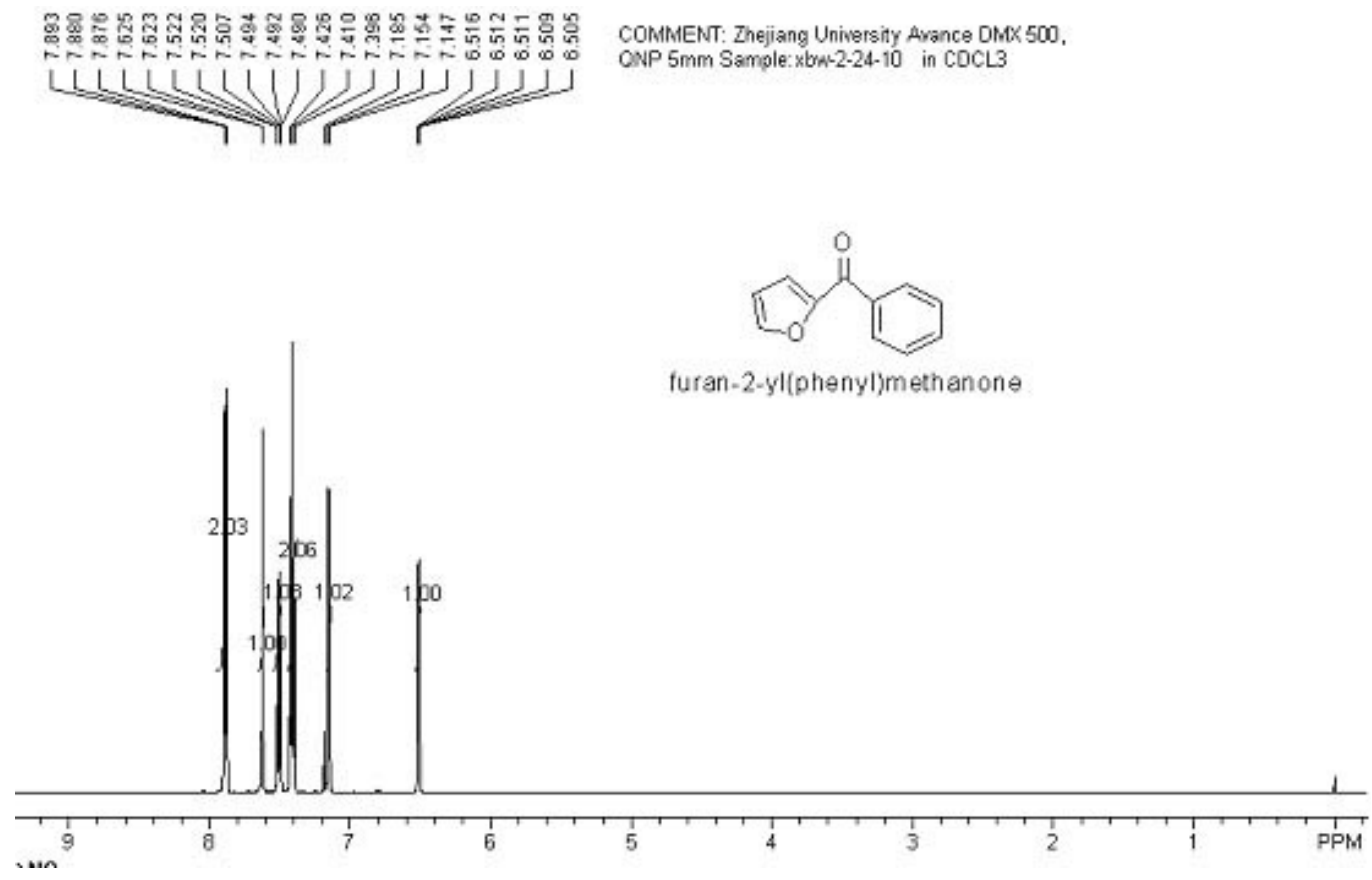
16.<smiles>CCCCCC(=O)c1ccccc1</smiles>

[T2-18, 942-92-7, Ref .11]

1-Phenylhexan-1-one: ${ }^{1} \mathrm{H}$ NMR (400MHz, $\left.\mathrm{CDCl}_{3}, \mathrm{TMS}\right)$ : $87.97-7.94(\mathrm{~m}, 2 \mathrm{H})$, 7.54-7.52 (t, $J=3.8 \mathrm{~Hz}, 1 \mathrm{H}), 7.47-7.43(\mathrm{q}, J=5.1 \mathrm{~Hz}, 2 \mathrm{H}), 2.97-2.93(\mathrm{t}, J=7.4 \mathrm{~Hz}, 2$ H), 1.81-1.72 (m, 2 H), 1.38-1.34 (m, 4 H), 0.93-0.89 (m, 3 H). MS (EI): m/z (\%): 176(M $\left.\mathrm{M}^{+}, 20\right), 133(10), 120(70), 105(100), 77(50), 51(10)$.
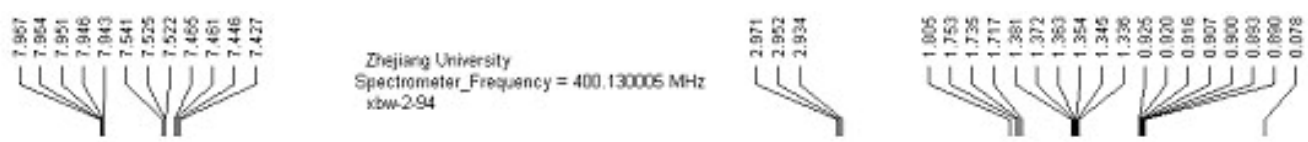

Thectrometer Fropiency $=400.130005 \mathrm{MH}$

xbm-2.94
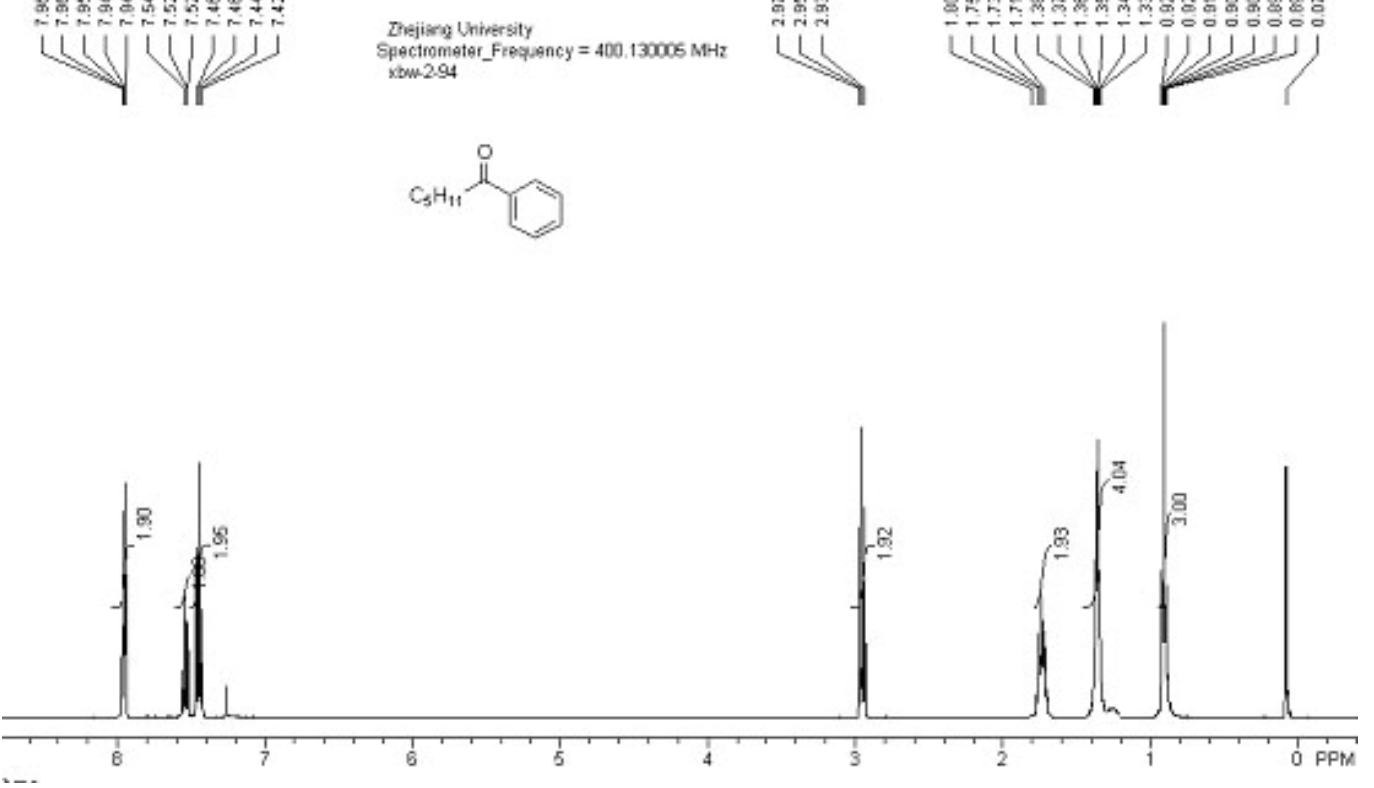
17.<smiles>O=C(Cc1ccccc1)c1ccccc1</smiles>

[T2-19, 451-40-1, Ref .15]

1, 2-Diphenylethanone: ${ }^{1} \mathrm{H}$ NMR (400MHz, $\mathrm{CDCl}_{3}, \mathrm{TMS}$ ): $\delta 8.03-8.00$ (q, $J=3.2 \mathrm{~Hz}$, $2 \mathrm{H}), 7.56-7.54(\mathrm{~d}, J=6.8 \mathrm{~Hz}, 1 \mathrm{H}), 7.48-7.46(\mathrm{q}, J=2.8 \mathrm{~Hz}, 2 \mathrm{H}), 7.44-7.43(\mathrm{~d}, J=0.8$ Hz, 2 H), 7.35-7.27 (m, 3 H), 4.29 (s, 2 H). MS (EI): m/z (\%): 196(M+, 50), 181(20), 165(5), 152(5), 119(100), 105(58), 91(40), 77(40), 65(20), 51(10).
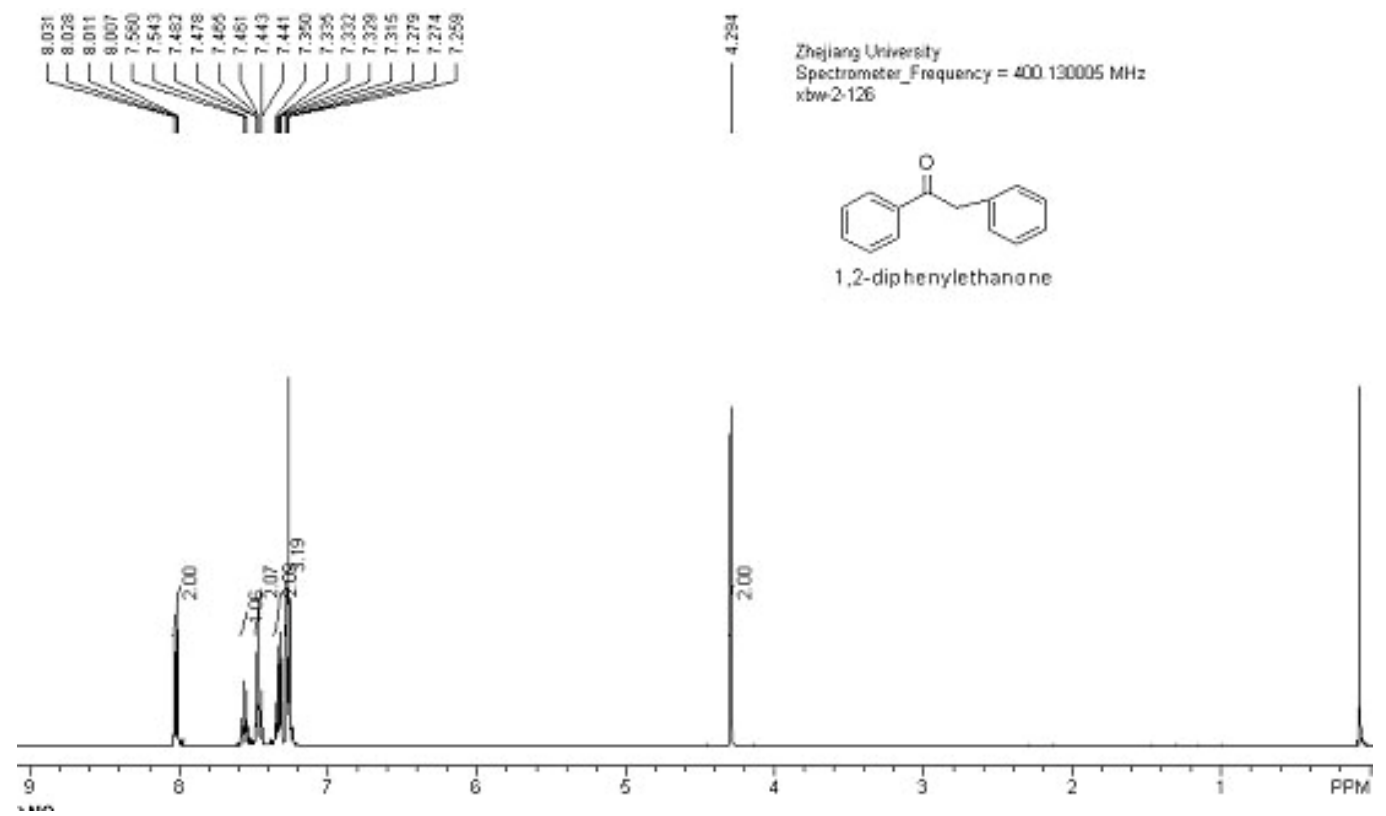
Reference

1. Park, S.; Kazlauskas, R. J. J. Org. Chem. 2001, 66, 8395-8401.

2. Urawa,Y.; Ogura, K. Tetrahedron Lett. 2003, 44, 271-273.

3. Wu, T. C.; Xiong, H. P.; Rieke, R. D. J. Org. Chem. 1990, 55, 5045-5051.

4. Goossen, L. J.; Ghosh, K. Eur. J. Org. Chem. 2002, 19, 3254-3267.

5. Kakino, R.; Narahashi, H.; Shimizu, I.; Yamamoto, A. Bull. Chem. Soc. Jpn. 2002, 75, 1333-1345.

6. Rahman, O.; Kihlberg, T.; Långström, B. Eur. J. Org. Chem. 2004, 3, 474-478.

7. Gmouh, S.; Yang, H.; Vaultier, M. Org. Lett. 2003, 5, 2219-2222.

8. Zhang, Y. D.; Rovis, T. J. Am. Chem. Soc. 2004, 126, 15964-15965

9. Rieke, R. D.; Kim, S. H.; Wu, X. J. Org. Chem.; 1997; 62(20); 6921-6927.

10. Wang, D. H.; Zhang, Z. G. Org. Lett., 2003, 5, 4645-4648.

11. Tatamidani, H.; Kakiuchi, F.; Chatani, N. Org. Lett. 2004, 6, 3597-3599.

12. Korn, Tobias J.; Knochel, Paul. Angew. Chem. Int. Ed. 2005, 44, 2947-2951.

13. Kazmierski, I.; Bastienne, M.; Gosmini, C.; Paris, J. M.; Périchon, J. J. Org. Chem., $200469,936-942$.

14. Kang, S. K.; Ryu, H. C.; Lee, S. W. J. Organomet. Chem. 2000, 610, 38-41.

15. Tatamidani, H.; Yokota K.; Kakiuchi, F.; Chatani, N. J. Org. Chem., 2004, 69, 5615-5621; 